\title{
Cultured enzyme-linked immunospot assay (ELISPOT) enhances detection of low HIV-specific immune responses in exposed seronegative individuals in Kenya Margaret Muturi*1, Charity Gichuki1 ${ }^{1}$, Gaudensia Mutua ${ }^{2}$ and Omu Anzala ${ }^{2}$
}

\author{
Address: ${ }^{1}$ Department of Biochemistry, Kenyatta University, P.O Box 43844-00100, Nairobi, Kenya and ${ }^{2}$ Department of Medical Microbiology, \\ Kenya Aids Vaccine Initiative, University of Nairobi, P.O Box 19676, Nairobi, Kenya \\ Email: Margaret Muturi* - mmuturi@kaviuon.org \\ * Corresponding author
}

from 2006 International Meeting of The Institute of Human Virology

Baltimore, USA. |7-2I November, 2006

Published: 21 December 2006

Retrovirology 2006, 3(Suppl I):P44 doi:I0.II86/I742-4690-3-SI-P44

(C) 2006 Muturi et al; licensee BioMed Central Ltd.

There is increasing evidence that many HIV exposed, seronegative individuals generate HIV-specific cellular immune responses. IFNg ELISPOT has emerged as one of the widely used assay to monitor HIV-specific immune responses. It is becoming the assay of choice for evaluation of HIV-vaccine-induced cell-mediated immune responses in many clinical trials. However, HIV specific IFNg T cell responses often wane with time and may eventually become undetectable using the direct ELISpot method. There is need for a more sensitive detection method to enhance the detection of such responses when they fall below levels detectable by direct ELISpot. This study seeks to determine if cultured ELISpot enhance detection of low HIV specific immune responses. 12 highrisk HIV negative individuals were followed up every 3 months for 9 months. During follow-up history was obtained, HIV rapid tests performed and blood samples obtained for assessment of HIV specific responses using both direct and cultured ELISpot assays. 5 discordant couples were also recruited and HIV specific responses assessed by both direct and cultured ELISpot. Preliminary data shows that there were no IFNg responses detected by direct ELISpot. 6/12 high-risk HIV seronegative volunteers gave positive responses to 1, 2, 4, 9 and 90 peptide pools using Cultured ELISpot at different time points during follow up. Positive responses range from 125 to 1934 spot forming units (SPU/106).
The results indicate that cultured ELISpot amplified the immune responses and thus enhanced their detection. It appears that cultured ELISpot is more sensitive than direct Elispot and it is the test of the future. 